\title{
24
}

\section{The PARSE Project}

\author{
I. E. Jelly ${ }^{l}$ and I. Gorton ${ }^{2}$
}

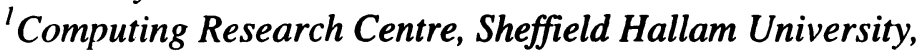

Sheffield S11 8HD, UK. Email: i.jelly@ shu.ac.uk

Tel : +44 (0)114253 3763, Fax: +44 (0)1142533161

${ }^{2}$ CSIRO Division of Information Technology, Locked Bag 17, North Ryde, NSW 2113, Australia.Email: iango@syd.dit.csiro.au

Tel: +61 (0)23253160, Fax: +61 (0)23253101

\begin{abstract}
Within the PARSE project, issues relating to the development of parallel and distributed software are being researched. These include analysis and design techniques, verification of system behaviour, performance evaluation and tool support. This paper reviews the work undertaken in the project, indicates future directions for research and provides a bibliography of key publications.
\end{abstract}

Keywords
parallel processing, distributed systems, software engineering, EPOCA, PARSE

\section{PROJECT AIMS}

The PARSE (PARallel Software Engineering) project began in 1991 as a collaborative project between Sheffield Hallam University and two Australian Universities. Its aim has been the development of techniques and tools to support the production of high quality software for a wide range of parallel and distributed systems. The collaboration has been extended to the Universities of Sheffield and Naples, and now includes an industrial partner.

Within the project, research has been focused on the issues involved in the development of parallel and distributed software systems. It brings together a number of projects which are addressing a range of technical issues within the field. These involve the development of appropriate techniques and tools to support good software engineering practice for parallel and distributed systems. The collaboration has revealed a unifying approach to the consideration of different aspects of the development of systems, based round the integration of pragmatic design engineering principles and formally based techniques.

Currently parallelism in software systems can be employed either to provide better performance in large scale scientific computations, or to support the development of smaller scale systems 
where issues of real time constraints, physically distributed control and fault tolerance are involved. In addition, system programmers constructing operating systems and associated low-level system utilities exploit concurrent tasks to share and manage resources amongst users and user programs. However the importance of parallel software is likely to grow in the next decade. Major technological advances in processing power, desktop operating systems, networking and userinterface systems will converge, creating new, high-volume application areas in distributed systems, consumer electronics and multimedia systems. All of these naturally lend themselves to parallel software solutions.

Within the PARSE project the emphasis had been on the development of design principles to promote the construction of efficient, robust and reusable parallel and distributed systems. A general framework is proposed to cover a range of application types and implementation environments. The designer may need to employ different views and techniques within the development process; the importance of providing clear mechanisms for interfacing with these has been recognised from the outset of the PARSE project, and the concept of methods integration is central to our approach. Although primarily aimed at software development, we are currently applying the techniques in relation to hardware/software codesign.

\section{PROJECT OUTCOMES}

The major outcomes from the project at this stage are:

1. Over twenty journal and international conference papers, and a number of technical reports.

2. Prototype CASE tools for the key phases of PARSE have been developed and demonstrated.

3. Techniques developed as part of the project are being employed for the production of industrial applications, thus providing feedback to the academic partners.

4. The collaborators have been active in the establishment of a research community in the area of parallel and distributed software engineering, and have organised workshops on this topic.

\section{KEY RESEARCH ISSUES}

\subsection{PARSE Methodology}

The PARSE methodology supports a systematic design refinement process and provides a graphical approach (PARSE process graphs) to express high level architecture and language independent design abstractions. A staged design methodology has been defined within which the developer moves from a high level abstract view of the system to a more concrete representation of the software. Different application types may require variations on the process: for example, real time systems design requires the introduction of temporal constraints at an early stage in the design process; for safety critical systems, formal verification may be required throughout the development phases.

\subsection{Design Notation}

The PARSE process graph notation allows developers to describe the system in terms of a hierarchy of interacting components. It is object-based and supports a staged design refinement approach. Process objects interact by message passing on designated communication paths. Both process objects and communication paths are classified according to their role in the system. In 
addition, information about the dynamic behaviour of the system is captured with the graphical notation by the introduction of path constructors which describe the order of handling of incoming messages by process objects. Process graphs can be systematically supplemented with additional behavioural details by using formalisms such as Petri nets or CSP, or a dedicated Behaviour Specification Language (BSL). Translation from BSL descriptions into Petri net specifications can be fully automated and forms the basis for the work within the EPOCA project.

\subsection{Tool Support}

Prototype design editors have been constructed to support developers in the construction of process graph/BSL designs, and work on more sophisticated versions of these is proceeding. The emphasis within this work is to ensure that the tools developed will support flexible integration of different techniques: appropriate interfaces must be defined to allow easy access to a range of verification and simulation and programming systems. Hence we are exploring the use of metaCASE systems to implement the PARSE support tools. In order to bridge between the design and implementation stages of the software development cycle, research into code generation for designs specified in BSL format has lead to the development of prototype code generators. Currently PVM and Occam tools have been implemented and code generators for DISC (Distributed C) under the EPOCA project are under consideration.

\subsection{EPOCA Integration}

Design validation plays an important role in the development of robust software systems, and the integration of behavioural analysis techniques involving Petri nets has been explored. Original work within the PARSE project demonstrated the potential for use of Petri net analysis, and recent collaboration with the EPOCA project team has provided a mechanism for this.

Under the EPOCA project, techniques have been developed for the analysis of parallel software systems by means of stochastic Petri nets. This resulted in the integration of the stochastic Petri net toolset GreatSPN with the DISC (DistributedC) programming environment. DISC allows distributed applications to be implemented and executed on a network of workstations. DISC programs can be automatically analysed by the use of GreatSPN.

Within the PARSE project further integration is planned. We have demonstrated how PARSE BSL descriptions can be translated either into DISC code, or directly to GreatSPN format. The design can therefore be subjected to analysis to provide initial feedback to the developer, and further analysed at the code stage. Work is currently underway to automate this translation and provide an interface to GreatSPN, a toolset based on stochastic Petri nets. This supports both qualitative and quantitative analysis, and introduces scope for performance prediction and modelling at the design stage.

\subsection{Client-Server Behaviour Modelling}

The production of deadlock free software is a primary consideration in the development of many parallel systems. Recent work has investigated how designs can be constrained to facilitate deadlock detection without having to explore the complete state space of the program's execution.. Client-server behaviour modelling involves the development of a design which conforms to certain guidelines with respect to the type of interactions permitted between process objects. The result is 
a software design which can be directly verified as deadlock free without recourse to analysis using a formalism such as Petri nets. A simple graphical notation has been developed to represent clientserver relationships within a software design. This notation can be used in association with PARSE process graphs, by mapping client-server behaviour graphs onto process graph descriptions to provide full design information for the developer. This approach would seem to be especially promising for complex, safety-critical systems.

\subsection{Object-Oriented Analysis Techniques}

The extent to which traditional analysis techniques offer a foundation for the development of parallel and distributed software systems forms the basis of our work in this area. Original work has suggested that object-oriented analysis techniques such as OMT could be used in association with PARSE design methods. We are now carrying out an evaluation of the role of different analysis techniques within the development framework for parallel and distributed systems.

\subsection{Hardware/Software Codesign}

Within the PARSE project we are researching techniques to support high level hardware/software codesign for a range of low power, hand held devices with stringent design constraints. Initial work has indicated that the PARSE design approach provides an appropriate framework for implementation independent design descriptions. Process graphs are used to specify the system architecture at a high level of abstraction; and BSL descriptions developed to describe the interactions between components. Translations can then performed from the BSL descriptions into appropriate hardware description languages, eg VHDL, and refined software design specifications. We are researching the introduction of constraint representation and partitioning strategies using process graphs and BSL.

\subsection{Design of Dynamic Distributed Systems}

The original PARSE process graph notation only supports dynamic process creation in a very limited manner, through the use of process object replication. However, for many applications for distributed computing platforms, this level of design specification is insufficient. We have therefore extended the core notation to cater for dynamic process object and communications path creation, and subsequent destruction. The extensions are currently being utilised and evaluated in the design of distributed interactive multimedia applications, with encouraging results.

\subsection{Case Studies and Industrial Trials}

In order to validate the PARSE approach, the methodology has been applied to a number of significant software developments, including a parallel logic language run-time support system, a database engine and a transport protocol for high speed networks. More recently the PARSE design method and process graph notation has been used in the development of an industrial real time embedded control system. Combined with the client-server modelling techniques it proved to be an effective and efficient method of construction deadlock free, concurrent software. In addition the notation was found to support team working by providing accessible design documentation. 


\section{FUTURE DIRECTIONS}

The following areas are currently under investigation:

Performance prediction: The development of appropriate simulation tools to support early performance prediction is under investigation. This work will complement the performance evaluation techniques available within the PARSE-EPOCA integration.

Client-server modelling: Future work is planned which will consider the application of these design techniques to a wide range of realistically sized systems. We shall explore the relationship with formal modelling techniques with a view to providing automated support for design verification within which the state explosion problem can be controlled.

High-level design abstractions: We are looking to develop high-level design templates that designers can reuse in different applications.

Designing for distributed object systems: Software development techniques for distributed systems has lead to the definition of different common object co-ordination models to support interaction between objects. We are looking at high level design techniques which allow designers to use these common object models in a secure and effective manner.

Training in parallel and distributed design practice: We are exploring the introduction of the high level design techniques developed during the PARSE project into both academic curricula and industrial training courses.

\section{SUMMARY AND COLLABORATION OPPORTUNITIES}

The PARSE project currently involves academic staff and students in a number of institutions and industrial partners. It is an open collaboration aimed at providing a forum for exploration of issues relating to parallel and distributed software development. The intention is to support a number of research directions within the project framework, building on existing co-operation to ensure that the high quality of the work is maintained.

We welcome further collaboration - both academic and industrial. We believe that there is a growing range of applications which demand the use of the type of techniques we have been investigating, and we are keen to explore their use in the development of real systems. More information can be found on the PARSE Web Page: http://www.dcs.shef.ac.uk/ prc/parse.html

\section{PARSE BIBLIOGRAPHY}

\subsection{Selected Journal and Conference Papers}

Gorton, I., Jelly, I.E. and Gray, J P (1993) PARSE: A Software Engineering Methodology for Parallel Program Design, in Proc IEEE Int Parallel Processing Symposium, April 1993, Newport Beach, CA, USA, IEEE Press

Donatelli, S., Franceschinis, G., Mazzocca, N. and Russo, S. (1994) Software Architecture of the EPOCA Integrated Environment in Proc 7th Int Conf on Modelling Techniques and Tools for Computer Performance Evaluation, May 1994, Vienna, Austria, Springer Verlag LNCS 794, pp 335-352, 
Jelly, I.E. and Gorton, I. (1994) Software Engineering for Parallel Systems in Information and Software Technology Journal, Vol. 36, No 7, pp 381-396

Knowles, C. and Collingwood, P. (1994) Parallel Software Development using an Object-Oriented Modelling Technique in Information and Software Technology Journal, Vol. 36, No 7, pp 397-404,

Gorton I., Jelly, I.E. and Chan, T.S. (1994) Engineering High Quality Parallel Software Using PARSE in Proc CONPAR, September 1994, Linz, Austria, Springer Verlag LNCS, pp 381-392

Birkinshaw, C.I. and Croll, P.R. (1995) Modelling the Client-Server Behaviour of Parallel Real-Time Systems Using Petri Nets" in Proc HICSS-28, (Hawaiian International Conference on System Sciences), January 1995, Maui, Hawaii, USA, IEEE Computer Society Press Vol. II, pp 339-348

Gorton, I., Jelly, I.E., Croll, P.R. and Nixon, P. (1995) Directions in Software Engineering for Parallel Systems in Proc HICSS-28 (Hawaiian International Conference on System Sciences) January 1995, Maui, Hawaii, USA, IEEE Computer Society

Gorton, I., Jelly, I.E. and Gray, J P. (1995) Object Based Modelling of Parallel Programs in IEEE Parallel and Distributed Technology Journal, Vol. 3, No. 2, 1995, IEEE Computer Society Press

Lloyd D.W., Jelly, I.E. and Cai, J. (1995) Evaluation of PARSE for High Level Codesign Specifications in Proc ICRAM '95 (Int Conf on Recent Advances in Mechatronics), August 1995, Istanbul, Turkey

Jelly, I.E., Croll, P.R., Birkinshaw, C.I. and Gorton, I. (1995) Client-Server Behaviour Modelling in PARSE in Proc Euromicro '95 Conference, Como, Italy, September 1995, IEEE Computer Society Press

Knowles, C., Jelly I.E. and Collingwood, P.C. (1995) Evaluation of Software Engineering Analysis Techniques for Parallel Software in Proc Euromicro 95 Conference, Como, Italy, September 1995, IEEE Computer Society Press

Jelly, I.E. and Gorton, I. (1995) Directions in CASE Technology for Parallel Software Development in Transputer Communications Journal, Vol. 3. No. 1, pp

Liu, A. and Gorton, I. (1996) Modelling Dynamic Distributed System Structures in PARSE in Proc 4th European Workshop on Parallel and Distributed Processing, January 1996, Braga, Portugal, IEEE Computer Society Press

Gorton, I., Jelly, I.E., Gray, J.P. and Chan, T.S. (1996) Reliable Parallel Software Construction using PARSE to appear in Concurrency: Practice and Experience Journal

Sadler, D.R., Lloyd, D.W. and Jelly, I.E. (1996) Object Based Hardware/Software Co-design to appear in Proc IEEE Int Conference on Computers and Communications, March 1996, Phoenix, USA, IEEE Computer Society Press

\subsection{Workshop Proceedings}

Workshop on "Software Engineering for Parallel Systems", Aachen, Germany, September 1993. Selected papers published in special edition of Information and Software Technology Journal, Vol. 36, No. 7, 1994. Guest editors, I.E. Jelly, I. Gorton and J. P. Gray'

Workshop on "CASE Technology for Parallel Systems Development", Como, Italy, September 1994. Selected papers in special edition of Transputer Communication Journal, Vol. 3, No. 1 (1995). Guest editors, I. E. Jelly and I. Gorton

Mini-track on "Software Engineering for Parallel Systems", in Proc HICSS-28 (Hawaiian International Conference on System Sciences), January 1995, Maui, Hawaii, USA, IEEE Computer Society 1995. Co-ordinators: I. Gorton, I. E. Jelly, P. R. Croll and P. Nixon 